\title{
Micropatterned composite membrane of polymerized and fluid lipid bilayers
}

\section{(Supporting Information)}

\author{
Kenichi Morigaki,\#* Kazuyuki Kiyosue, \# Takahisa Taguchi \# \\ Special Division for Human Life Technology, National Institute of Advanced Industrial \\ Science and Technology (AIST), Ikeda 563-8577, Japan \\ \# Current affiliation: Research Institute for Cell Engineering, AIST
}

Fax: +81-72-751-9628

e-mail: morigaki-kenichi@aist.go.jp 
UV/visible absorption spectroscopy

Photopolymerization of DiynePC resulted in formation of a conjugated polymer backbone and could be most conveniently monitored by UV/ visible absorption spectroscopy. UV/ visible absorption spectra were obtained by using a Shimadzu UV-3150 spectrophotometer (Shimadzu, Kyoto, Japan). The samples were dried by nitrogen before the measurements. This procedure did not affect the absorption spectra of the poly-diacetylene films (the obtained spectra were identical with the in situ measurements). Figure s1 (A) shows the UV/ visible absorption spectra of the monomeric and polymerized bilayers of DiynePC. The polymeric bilayer has an absorption band between $400 \mathrm{~nm}$ and $550 \mathrm{~nm}$ that originates from conjugated ene-yne backbones. The peak area of this band is plotted in Figure s1 (B) as a function of the UV irradiation dose applied for the polymerization. The absorption measured directly after the photopolymerization (filled symbols) increased with the applied UV dose up to $1.5 \mathrm{~J} / \mathrm{cm}^{2}$ and decreased gradually for a larger UV dose. The decrease in absorption peak area does not necessarily mean degradation or decomposition of the polymeric bilayers, however, because any conformational changes that disrupt the effective conjugation of polymeric backbones can reduce the absorption peak areas. In the lithographic photopolymerization non-reacted monomers are removed by immersing the 
sample in $0.1 \mathrm{M}$ SDS solution. The UV/ visible absorption spectra after the SDS treatment are plotted as open symbols. The treatment resulted in a considerable reduction of the absorption peak area, especially for small UV irradiation doses (less than ca. $1 \mathrm{~J} / \mathrm{cm}^{2}$ ). On the other hand, a substantial portion of the absorption band remained after the SDS treatment, if the polymers were created with larger UV doses. The higher retention of the peak area indicates that the polymerized bilayer became more solubilization-resistant and the bilayer structure also acquired an enhanced robustness against the disordering effect of SDS.
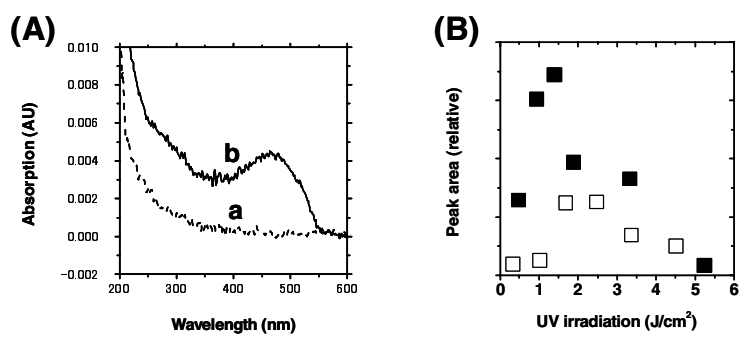

Figure s1 UV/ visible absorption spectroscopy of polymerized DiynePC: (A) UV/ visible absorption spectra of a bilayer on quartz substrate (a) before and (b) after the polymerization. (B) The peak area of the polymer adsorption band was plotted as a function of the UV irradiation dose without (filled symbols) and with (open symbols) the treatment in $0.1 \mathrm{M}$ SDS $\left(30\right.$ minutes at $\left.25^{\circ} \mathrm{C}\right)$. 